\title{
Technical Aspects \\ for the Evaluation of Circulating Nucleic Acids (CNAs): Circulating Tumor DNA (ctDNA) and Circulating MicroRNAs
}

\author{
M. Castiglia, A. Perez, M.J. Serrano, M. Ciaccio, \\ V. Bazan, and Antonio Russo
}

Circulating nucleic acids (CNAs), for example, circulating tumor DNA (ctDNA) and circulating microRNA (miRNA), represent promising biomarkers in several diseases including cancer. They can be isolated from many body fluids, such as blood, saliva, and urine. Also ascites, cerebrospinal fluids, and pleural effusion may be considered

M. Castiglia and A. Perez contributed equally to this work.

M. Castiglia $\bullet$ A. Perez

Department of Surgical, Oncological and Oral Sciences, Section of Medical Oncology, University of Palermo, Via del Vespro 129, 90127 Palermo, Italy

\section{M.J. Serrano}

GENYO, Centre for Genomics and Oncological Research (Pfizer/University of Granada/ Andalusian Regional Government),

PTS Granada Av. de la Ilustración, 114-18016 Granada, Spain

M. Ciaccio

Section of Clinical Biochemistry and Clinical Molecular Medicine, Department of Biopathology and Medical Biotechnology, University of Palermo U.O.C. Laboratory Medicine - CoreLab, Policlinico University Hospital, Palermo, Italy

V. Bazan • A. Russo, MD, PhD ( $\bowtie)$

Department of Surgical, Oncological and Oral

Sciences, Section of Medical Oncology, University of Palermo, Via del Vespro 129, 90127 Palermo, Italy

Institute for Cancer Research and Molecular Medicineand Center of Biotechnology, College of Science and Biotechnology, Philadelphia, PA, USA e-mail: antonio.russo@usa.net as a source of CNAs, but with several and intrinsic limitations. Therefore, blood withdrawal represents one of the best sources for CNAs due to the very simple and minimally invasive way of sampling. Moreover, it can be repeated at different time points, giving the opportunity for a real-time monitoring of the disease.

CNAs are spread from both cancer and normal cells, but in cancer patients their concentrations are greater $[1,2]$. Nevertheless, the mechanisms underlying their release are not fully understood. Some evidences show that CNAs can be released through a passive mechanism; indeed, infiltrating phagocytes clear apoptotic or necrotic cells under normal physiologic circumstances. This does not happen efficiently within the tumoral mass, leading to the accumulation of cellular debris and its inevitable release into the circulation. Another possible way of CNAs release could be through extracellular vesicles, such as exosomes. In this case, CNAs are packed inside exosome and actively secreted by cells. This seems to be more realistic for miRNAs, whereas for DNA there are still conflicting data.

\section{Circulating Tumor DNA (ctDNA)}

Circulating cell-free DNA (cfDNA) is highly fragmented, and therefore it represents a challenging analyte. It has been shown that the length 
of cfDNA strands is often between 200 and 180 base pairs, suggesting that apoptosis likely produces the majority of cfDNA in circulation [3]. Circulating tumor DNA (ctDNA) is part of the cfDNA deriving from the tumor mass. The easiest way to identify the ctDNA is to investigate the presence of somatic driver mutations, which, by definition, can be exclusively found on tumor. Nevertheless, several methods have shown that the fraction of ctDNA varies greatly, between $0.01 \%$ and more than $90 \%$ [3]. Moreover, different tumor types do not release the same amount of ctDNA, and, even in patients with the same disease, the concentration of ctDNA may vary consistently [4].

Several pre-analytical variables, such as blood collection and handling, ctDNA extraction protocols, and storage temperature may affect the quantity and quality of ctDNA fragments in a sample [5-8]. As previously mentioned, blood represents the most used source for ctDNA. Nevertheless, there is a big question: serum or plasma?

In the majority of clinical trails, EDTA containing tubes are used for blood collection (4-9 [9]). Using these tubes clotting is inhibited, and thus it is possible to recover plasma that represent the matrix of choice for ctDNA extraction. Actually also serum can be used as a matrix to isolate ctDNA; indeed, it has been reported that the amount of ctDNA in serum can be 2-24 times higher than in plasma. This can be a consequence of the clotting process that causes white blood cells (WBCs) breaking, finally leading to the release of wild-type DNA. This contamination causes a further dilution of the tumor-specific DNA, making it even more difficult to detect. However, it has been reported that in some cases it might be advantageous to analyze both serum and plasma, as this increases the chances to detect the specific mutation [10].

Another important pre-analytical aspect is the time that elapses between the withdrawal and its processing for plasma recovery. Indeed, the more the time passes, the more is the risk of WBCs lysis, leading again to ctDNA contamination with wild-type background DNA. Moreover ctDNA is associated with a high turnover (15 min halflife), and therefore after blood collection, it is recommended to proceed with plasma preparation by centrifugation within $1 \mathrm{~h}$ [11]. Plasma can be stored for a long period at $-20{ }^{\circ} \mathrm{C}$ or immediately processed for ctDNA extraction.

ctDNA extraction can be performed through different kits; recently, Sorber L et al. [12] have compared the isolation efficiency of the most used kit, the QIAamp circulating nucleic acid kit (QIA), with four other cfDNA isolation kits: the PME free-circulating DNA Extraction Kit (PME), the Maxwell RSC ccfDNA Plasma Kit (RSC), the EpiQuick Circulating Cell-Free DNA Isolation Kit (EQ), and two consecutive versions of the NEXTprep-Mag cfDNA Isolation Kit $\left(\mathrm{NpM}_{\mathrm{V} 1 / 2}\right)$. A total of ten samples were used, and five of them harbored KRAS mutations. In the study, the detection of KRAS mutation and total cell-free DNA concentration were performed with droplet digital PCR, whereas real-time PCR was used to evaluate cfDNA integrity. They showed that QIA and the RSC kits displayed similar isolation efficiencies, whereas the yield generated by the PME and $\mathrm{NpM}_{\mathrm{V} 2}$ kits was significantly lower [12]. Interestingly, Sonnenberg et al. developed an electrokinetic technique that allowed rapid isolation of cfDNA directly from blood [13, 14].

Following extraction, another important issue is the quantification method. There is no standardization of the quantification method, which can lead to different results. The most commonly used techniques include spectrophotometric methods, fluorescent dyes, or quantitative PCR-based methods [15]. The identification of a reliable and efficient method for cfDNA quantification is fundamental for the clinical evaluation of ctDNA as a liquid biopsy in order to obtain consistent data, comparable between laboratories.

Plasma DNA investigation can be achieved through two different analytical approaches: a targeted approach and an untargeted approach (Fig. 6.1). The targeted approach relies on the possibility to analyze known genetic mutations that occurs in hotspot region of specific genes with implications for therapy decisions; this is the case, for example, of KRAS, EGFR, and BRAF genes in lung, colon, and melanoma tumors, respectively. Among these methods, we can include real-time PCR; digital PCR (dPCR); 


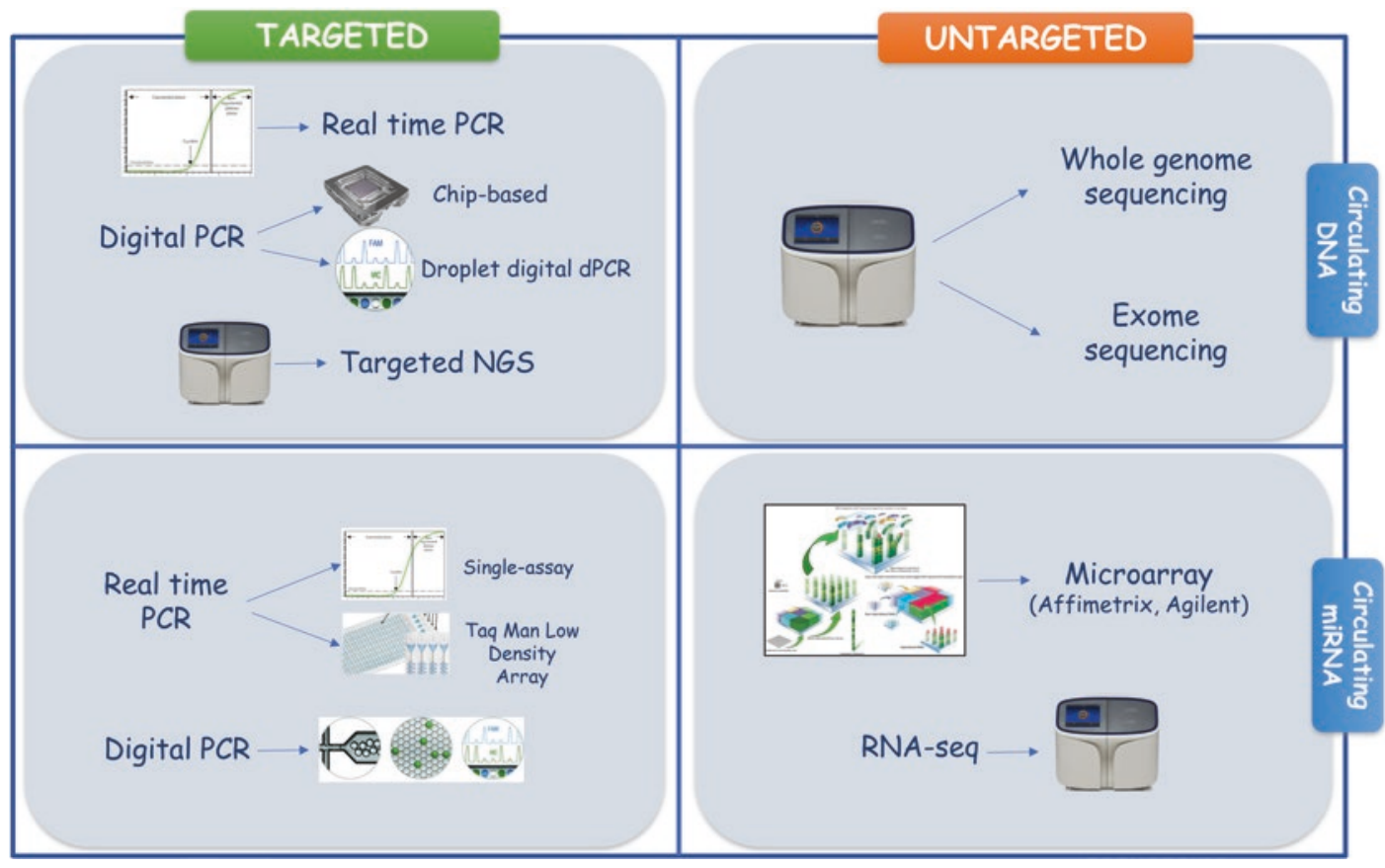

Fig. 6.1 Targeted and untargeted approaches for circulating DNA and circulating miRNAs evaluation

droplet digital PCR (ddPCR); beads, emulsions, amplification, and magnetics (BEAMing); and targeted next-generation sequencing (NGS).

In the untargeted approach, it is possible to investigate ctDNA without the knowledge of any specific mutations present in the primary tumor. This can be achieved through whole genome sequencing using NGS platforms. Nevertheless, this analysis is quite expensive and sometimes difficult to interpret; thus, it can be used for biomarkers discovery in the context of disease monitoring, detection of molecular resistance, and identification of new therapeutic targets. Despite whole genome sequencing, a more cost-effective method in the exome sequencing, which does not require prior knowledge of the genetic landscape of the tumor.

As previously mentioned, the main targeted approaches are real-time PCR, dPCR, ddPCR, BEAMing [16], and targeted NGS. Real-time PCR represents the oldest technique, but its sensitivity has been dramatically improved thanks to the introduction of the ARMS technology (amplification refractory mutation system) [17, 18]. Nevertheless, the power of this technique in detecting mutant allele at a very low frequency is limited, and therefore other more sophisticated methods have been developed. Through the dPCR approach, the DNA sample is partitioned into thousands of single PCR reactions, improving detection power [19]. In ddPCR, the partitioning is obtained through an emulsion PCR, each generated droplets ideally represent a PCR reactor. At the end of the analysis, software allows to identify a positive or a negative signal indicating the presence or absence of a target sequence. Therefore, a mutated ctDNA can be detected in a wide background of wild-type sequences. The dPCR platforms now available are various, each of them with a more or less different workflow, but they all share a very high sensitivity [20].

NGS is emerging as a very interesting technique because it has revolutionized our approach to molecular testing, indeed we can analyze multiple genes and multiple patients at a time with a consistent reduction in time and money. Of great interest, there is the paper of Newman et al. that has developed cancer personalized profiling by deep sequencing (CAPP-Seq) [21]. CAPP-Seq 
method is able to detect ctDNA in $100 \%$ of patients with stage II-IV non-small-cell lung carcinoma and in $50 \%$ of patients with stage I. The diagnostic specificity was $96 \%$ for mutant allele fractions down to approximately $0.02 \%$ [21].

\section{Circulating MicroRNA}

The promising role of circulating miRNAs as disease biomarkers has been deeply evaluated and still continues to increase the interest of scientists. However, the technical aspects of miRNAs isolation, measurement, and quantification still represent the critical steps of circulating miRNAs analysis. Indeed, sample processing, isolation, hemolysis in blood samples, the lack of stable reference gene, and the wide variety of genome platforms are only a few of the many not negligible aspects [22].

In circulating miRNAs analysis, the first and pivotal step is to identify a feasible source of nucleic acids. As reported in the study of Weber et al., the most common source of circulating miRNAs are plasma, serum, urine, and saliva but also microvesicles and exosomes [23]. Even if the exosomal miRNAs can probably provide more information, their isolation is complex [24]. The isolation of circulating miRNAs from plasma or serum is easier despite the high content of blood components in these body fluids. Furthermore, plasma and serum specimens often show a different spectrum of miRNAs also within the same individuals. Serum seems to be better source for miRNA isolation because the yield of miRNA is greater than the one obtained from plasma; this is probably due to the contamination of RNAs deriving from platelets during the clotting process [25]. Also in plasma, the levels of miRNA could be influenced by hemolysis as recently reported by Kirschner et al. In fact, miR-16 and miR-451 plasma levels are highly increased as usually they are in blood cells $[26,27]$. Generally, the concentration of miRNAs in body fluids is very low. Therefore, the isolation and enrichment of miRNAs is an extremely delicate and important procedure. Nowadays, for the RNAs isolation we can rely on manual extraction methods such as the phenol/chloroform or commercially distributed kits. Overall, they show differential efficiency even if the phenol/chloroform method showed higher yields (400 ng/500 uL of plasma) if compared to the commercial kits $(50 \mathrm{ng} / 200 \mathrm{uL}$ of plasma) [28, 29]. Given the low representation of miRNAs in body fluids, another crucial step is represented by quantification. To date, many different quantitative approaches have been tested to analyze circulating miRNAs. One of the most commonly used approaches for the quantification of a specific miRNA is quantitative real-time PCR (qRT-PCR). It can rely on two different strategies:

1. Relative quantification: the relative expression of a specific miRNA is measured by comparing its level with the expression levels of a reference endogenous gene. Unfortunately, the debate on the most reliable endogenous miRNA is still open. Indeed, some groups speculate on the high reliability of miR-16, which expression levels are highly stable in different tissues, while some others demonstrated inconsistent expression of miR-16 in plasma and serum [30, 31]. However, a combination of several genes among all those selected seems to be the best approach to follow [32].

2. Absolute quantification: this method relies on the generation of a standard curve. The results of absolute quantification are often indicated as copies per $\mathrm{uL}$ of plasma or serum. In the last years, the introduction of digital technologies (dPCR, ddPCR) has deeply increased the sensibility of the standard PCR approaches. Indeed, without the aid of a standard curve, PCR-positive and PCR-negative reactions are counted and then the result is converted as number of copies of the specific target.

Regarding the expression profile of circulating miRNAs, the most commonly used platform is TaqMan Low Density Array (TLDA, ThermoFisher) based on qRT-PCR. This highsensitive platform allows analyzing up to 754 miRNAs at the same time. Generally, the 382-well format is the most developed for its reduced costs, 
high throughput, and simple workflow. Moreover, its high sensibility allows the use of a low input of RNA (1-500 ng) [33, 34]. Another platform used for miRNAs profile is Microarray technology. Microarray is based on the hybridization of nucleic acids on different supports and for its less sensitivity, generally requires a higher RNA input (100 nglug) that probably represents the major limitation of this application. Moreover, it can often be difficult to discriminate mature from immature miRNAs forms due to background and cross-hybridization issues [35, 36]. The recent introduction of deep sequencing miRNAs (miRNA-seq), a NGS approach, allowed not only to assess miRNA expression levels but also to identify unknown miRNAs. The major limitation of using routinely NGS is strictly correlated to its high costs as well as time consuming. Moreover, it generally requires big amount of input RNA even if there are attempts to work with less starting material (5 ng). Nowadays, the most popular NGS technology used for circulating miRNAs analysis is Solexa sequencing by Illumina [37, 38]. Recently, a novel technology combining serial analysis of gene expression (SAGE) with NGS technology has been developed. The so-called digital gene expression (DGE) allows to simultaneously study novel potential miRNAs and analyze their expression level [22]. In conclusion, the choice of the proper platform to analyze circulating miRNAs strictly depends on the aim and conditions of the study.

\section{References}

1. Delgado PO, Alves BC, Gehrke FS, et al. Characterization of cell-free circulating DNA in plasma in patients with prostate cancer. Tumour Biol. 2013;34:983-6.

2. Hashad D, Sorour A, Ghazal A, Talaat I. Free circulating tumor DNA as a diagnostic marker for breast cancer. J Clin Lab Anal. 2012;26:467-72.

3. Diaz LA Jr, Bardelli A. Liquid biopsies: genotyping circulating tumor DNA. J Clin Oncol. 2014;32(6):579-86. doi:10.1200/JCO.2012.45.2011. Epub 2014 Jan 21.

4. Bettegowda C, Sausen M, Leary RJ, et al. Detection of circulating tumor DNA in early- and late-stage human malignancies. Sci Transl Med. 2014;6:224ra224.
5. Umetani N, Kim J, Hiramatsu S, et al. Increased integrity of free circulating DNA in sera of patients with colorectal or periampullary cancer: direct quantitative PCR for ALU repeats. Clin Chem. 2006;52:1062-9.

6. Chan KC, Yeung SW, Lui WB, et al. Effects of preanalytical factors on the molecular size of cell-free DNA in blood. Clin Chem. 2005;51:781-4.

7. Swinkels DW, Wiegerinck E, Steegers EA, de Kok JB. Effects of blood-processing protocols on cellfree DNA quantification in plasma. Clin Chem. 2003;49:525-6.

8. Chiu RW, Poon LL, Lau TK, et al. Effects of blood-processing protocols on fetal and total DNA quantification in maternal plasma. Clin Chem. 2001;47:1607-13.

9. Malapelle U, Pisapia P, Rocco D, Smeraglio R, di Spirito M, Bellevicine C, Troncone G. Next generation sequencing techniques in liquid biopsy: focus on non-small cell lung cancer patients. Transl Lung Cancer Res 2016;5(5):505-510.

10. Karachaliou N, Mayo-de las Casas C, Queralt C, et al. Association of EGFR L858R mutation in circulating free DNA with survival in the EURTAC trial. JAMA Oncol. 2015;1:149-57.

11. Malapelle U, Pisapia P, Rocco D, et al. Next generation sequencing techniques in liquid biopsy: focus on non-small cell lung cancer patients. Transl Lung Cancer Res. 2016;5:505-10.

12. Sorber L, Zwaenepoel K, Deschoolmeester V, et al. A comparison of cell-free DNA isolation kits: isolation and quantification of cell-free DNA in plasma. $\mathrm{J}$ Mol Diagn. 2017;19:162-8.

13. Sonnenberg A, Marciniak JY, Rassenti L, et al. Rapid electrokinetic isolation of cancer-related circulating cell-free DNA directly from blood. Clin Chem. 2014;60:500-9.

14. Sonnenberg A, Marciniak JY, Skowronski EA, et al. Dielectrophoretic isolation and detection of cancerrelated circulating cell-free DNA biomarkers from blood and plasma. Electrophoresis. 2014;35:1828-36.

15. Devonshire AS, Whale AS, Gutteridge A, et al. Towards standardisation of cell-free DNA measurement in plasma: controls for extraction efficiency, fragment size bias and quantification. Anal Bioanal Chem. 2014;406:6499-512.

16. Bidard FC, Madic J, Mariani P, et al. Detection rate and prognostic value of circulating tumor cells and circulating tumor DNA in metastatic uveal melanoma. Int J Cancer. 2014;134:1207-13.

17. Spindler KL, Pallisgaard N, Vogelius I, Jakobsen A. Quantitative cell-free DNA, KRAS, and BRAF mutations in plasma from patients with metastatic colorectal cancer during treatment with cetuximab and irinotecan. Clin Cancer Res. 2012;18:1177-85.

18. Spindler KL, Pallisgaard N, Andersen RF, Jakobsen A. Changes in mutational status during third-line treatment for metastatic colorectal cancer - results of consecutive measurement of cell free DNA, KRAS and BRAF in the plasma. Int J Cancer. 2014;135:2215-22. 
19. Taly V, Pekin D, Benhaim L, et al. Multiplex picodroplet digital PCR to detect KRAS mutations in circulating DNA from the plasma of colorectal cancer patients. Clin Chem. 2013;59:1722-31.

20. Sorber L, Zwaenepoel K, Deschoolmeester V, et al. Circulating cell-free nucleic acids and platelets as a liquid biopsy in the provision of personalized therapy for lung cancer patients. Lung Cancer. 2016;107:100.

21. Newman AM, Bratman SV, To J, et al. An ultrasensitive method for quantitating circulating tumor DNA with broad patient coverage. Nat Med. 2014;20:548-54.

22. Hruštincová A, Votavová H, Dostálová MM. Circulating MicroRNAs: methodological aspects in detection of these biomarkers. Folia Biol (Praha). 2015;61:203-18.

23. Weber JA, Baxter DH, Zhang S, et al. The microRNA spectrum in 12 body fluids. Clin Chem. 2010;56:1733-41.

24. Hunter MP, Ismail N, Zhang X, et al. Detection of microRNA expression in human peripheral blood microvesicles. PLoS One. 2008;3:e3694.

25. Fang C, Zhu DX, Dong HJ, et al. Serum microRNAs are promising novel biomarkers for diffuse large B cell lymphoma. Ann Hematol. 2012;91:553-9.

26. Kirschner MB, Kao SC, Edelman JJ, et al. Haemolysis during sample preparation alters microRNA content of plasma. PLoS One. 2011;6:e24145.

27. Cheng HH, Yi HS, Kim Y, et al. Plasma processing conditions substantially influence circulating microRNA biomarker levels. PLoS One. 2013;8:e64795.

28. Monleau M, Bonnel S, Gostan T, et al. Comparison of different extraction techniques to profile microRNAs from human sera and peripheral blood mononuclear cells. BMC Genomics. 2014;15:395.

29. Kroh EM, Parkin RK, Mitchell PS, Tewari M. Analysis of circulating microRNA biomarkers in plasma and serum using quantitative reverse transcription-PCR (qRT-PCR). Methods. 2010;50:298-301.

30. Xiang M, Zeng Y, Yang R, et al. U6 is not a suitable endogenous control for the quantification of circulating microRNAs. Biochem Biophys Res Commun. 2014;454:210-4.

31. Filková M, Aradi B, Senolt L, et al. Association of circulating miR-223 and miR-16 with disease activity in patients with early rheumatoid arthritis. Ann Rheum Dis. 2014;73:1898-904.

32. Chen X, Liang H, Guan D, et al. A combination of let-7d, let-7g and let-7i serves as a stable reference for normalization of serum microRNAs. PLoS One. 2013;8:e79652.

33. Ge Q, Zhou Y, Lu J, Bai Y, Xie X, Lu Z. miRNA in plasma exosome is stable under different storage conditions. Molecules. 2014;19(2):1568-75.

34. Zearo S, Kim E, Zhu Y, Zhao JT, Sidhu SB, Robinson BG, Soon PSh. MicroRNA-484 is more highly expressed in serum of early breast cancer patients compared to healthy volunteers. BMC Cancer 2014; 14:200.

35. Steudemann C, Bauersachs S, Weber K, Wess G. Detection and comparison of microRNA expression in the serum of Doberman Pinschers with dilated cardiomyopathy and healthy controls. BMC Vet Res. 2013;9:12.

36. Blenkiron C, Askelund KJ, Shanbhag ST, et al. MicroRNAs in mesenteric lymph and plasma during acute pancreatitis. Ann Surg. 2014;260:341-7.

37. Creighton CJ, Reid JG, Gunaratne PH. Expression profiling of microRNAs by deep sequencing. Brief Bioinform. 2009; 10:490-7.

38. Wang Z, Gerstein M, Snyder M. RNA-Seq: a revolutionary tool for transcriptomics. Nat Rev Genet. 2009;10:57-63. 